Canadian Science Publishing

Applied Physiology, Nutrition, and Metabolism Physiologie appliquée, nutrition et métabolisme

\title{
The effect of exercise intensity and excess post-exercise oxygen consumption on postprandial blood lipids in physically-inactive men
}

\begin{tabular}{|c|c|}
\hline Journal: & Applied Physiology, Nutrition, and Metabolism \\
\hline Manuscript ID & apnm-2016-0581.R3 \\
\hline Manuscript Type: & Article \\
\hline Date Submitted by the Author: & 15-Mar-2017 \\
\hline Complete List of Authors: & $\begin{array}{l}\text { Littlefield, Laurel; Lubbock Christian University, Exercise and Sport } \\
\text { Sciences } \\
\text { Papadakis, Zacharias; Baylor University, School of Education, Department } \\
\text { of Health, Human Performance and Recreation } \\
\text { Rogers, Katie; Baylor University, School of Education, Department of } \\
\text { Health, Human Performance and Recreation } \\
\text { Moncada-Jiménez, José; University of Costa Rica, Human Movement } \\
\text { Sciences } \\
\text { Taylor, J. Kyle; Auburn University Montgomtery, Medical \& Clinical } \\
\text { Laboratory Sciences } \\
\text { Grandjean, Peter W.; Baylor University }\end{array}$ \\
\hline \multicolumn{2}{|l|}{$\begin{array}{r}\text { Is the invited manuscript for } \\
\text { consideration in a Special } \\
\text { Issue? : }\end{array}$} \\
\hline Keyword: & $\begin{array}{l}\text { Excess Post-Exercise Oxygen Consumption, Triglycerides, Postprandial } \\
\text { Lipemia, High-Intensity Exercise, exercise intensity < exercise }\end{array}$ \\
\hline
\end{tabular}

\section{SCHOLARONE" \\ Manuscripts}




\section{Title}

The effect of exercise intensity and excess post-exercise oxygen consumption on postprandial blood lipids in physically-inactive men

\section{Authors}

Laurel A. Littlefield, Zacharias Papadakis, Katie M. Rogers, José Moncada-Jiminez, J. Kyle Taylor, Peter W. Grandjean

\section{Corresponding Author}

Laurel A. Littlefield, Exercise and Sport Sciences, Lubbock Christian University, $560119^{\text {th }}$

Street, Lubbock, TX, 79407, 806-720-7865, laurel.littlefield@1cu.edu

\section{Author Affiliations}

LAL ${ }^{1}$, ZP, KMR, PWG: College of Health \& Human Sciences, HHPR, One Bear Place \#97313, Baylor University, Waco, TX 76798; laurel.littlefield@1cu.edu, Zach_Papadakis@baylor.edu, katie.rogers@ucdenver.edu,Peter_Grandjean@baylor.edu

JMJ: Department of Human Movement Sciences, Human Movement Sciences Research Center, University of Costa Rica, San José, Costa Rica, P.O. Box 239-1200; jose.moncada@ucr.ac.cr JKT: Medical \& Clinical Laboratory Sciences, Auburn University Montgomery, P.O. Box 244023, Montgomery, AL, 36124; jtaylor@aum.edu

${ }^{1}$ LAL is currently affiliated with Lubbock Christian University, $560119^{\text {th }}$ Street, Lubbock TX, 79407, laurel.littlefield@1cu.edu 


\section{ABSTRACT}

Background: Reductions in postprandial lipemia have been observed following aerobic exercise of sufficient energy expenditure. Increased excess post-exercise oxygen consumption (EPOC) has been documented when comparing high- versus low-intensity exercise. The contribution of EPOC energy expenditure to alterations in postprandial lipemia has not been determined.

Objective: The purpose of this study was to evaluate the effects of low- and high-intensity exercise on postprandial lipemia in healthy, sedentary, overweight and obese men $(43+10$ years; $31.1+7.5 \mathrm{ml} / \mathrm{kg} / \mathrm{min} ; 31.8+4.5 \mathrm{~kg} / \mathrm{m}^{2}$ ) and to determine the contribution of EPOC to reductions in postprandial lipemia.

Design: Participants completed 4 conditions: non-exercise control, low-intensity exercise at 40$50 \% \mathrm{VO}_{2} \mathrm{R}(\mathrm{LI})$, high-intensity exercise at 70-80\% $\mathrm{VO}_{2} \mathrm{R}(\mathrm{HI})$, and $\mathrm{HI}$ plus EPOC re-feeding (HI + EERM) where the difference in EPOC energy expenditure between LI and HI was re-fed in the form of a sports nutrition (Power Bar ${ }^{\circledR}$ ) bar. Two hours following exercise participants ingested a high-fat (1,010 kcals, 99g sat fat) test meal. Blood samples were obtained before exercise, before the test meal, and at 2-, 4- and 6- hours postprandially.

Results: Triglyceride incremental area-under-the-curve $\left(\mathrm{AUC}_{\mathrm{I}}\right)$ was significantly reduced following LI, HI and HI + EERM when compared to non-exercise control $(p<0.05)$ with no differences between the exercise conditions $(p>0.05)$.

Conclusions: Prior LI and HI exercise equally attenuated postprandial triglyceride responses to the test meal. The extra energy expended during EPOC does not contribute significantly to exercise energy expenditure or to reductions in postprandial lipemia in overweight men. Key Words: Postprandial Lipemia, Postprandial Blood Lipids, Triglycerides, Excess-Post Exercise Oxygen Consumption (EPOC), Exercise Intensity, High-Intensity Exercise 
Exercise Intensity and Postprandial Lipemia

\section{INTRODUCTION}

Exaggerated elevations in postprandial triglycerides are associated with increased risk for the development of cardiovascular disease (CVD) and are observed in coronary heart disease (CHD), hypertension, and metabolic syndrome (MetS) (Bansal, et al., 2007, Karpe, et al., 1999, Kolovou, et al., 2003, Nordestgaard, et al., 2007, Patsch, et al., 1992). High plasma triglycerides are associated with increased triglyceride-rich lipoprotein remnants (TRL), small, dense lowdensity lipoprotein cholesterol (LDLC) and oxidized LDLC, and are inversely associated with high-density lipoprotein cholesterol (HDLC) levels (Kathiresan, et al., 2006, Kolovou, et al., 2011, Park, et al., 2011, Zilversmit, 1995). Collectively, these atherogenic lipid abnormalities promote the development of CVD.

Aerobic exercise performed 1 to 16 hours prior to meal ingestion reduces postprandial lipemia (Aldred, et al., 1994, Gill, et al., 1998, Petitt and Cureton, 2003, Zhang, et al., 2004). The positive effect of exercise in mitigating postprandial lipemia has been observed following high-fat or mixed test meals with varying macronutrient composition. The fat content of individual test meals has ranged from approximately $35-90 \%$ of the total calories consumed in one sitting (Burton, et al., 2008, Gill, et al., 1998, Kolovou, et al., 2011, Zhang, et al., 2004). Gill, et.al, (1998) was the first to suggest that the positive effect of exercise on postprandial lipemia is mediated, in part, by the energy expenditure of the exercise session. Exercise of varying intensities and durations yield similar significant reductions in postprandial triglycerides when sessions are isocaloric (Mestek, et al., 2008, Tsetsonis and Hardman, 1996). Furthermore, exercise sessions that elicit greater caloric expenditure, by increased intensity or duration, enhance reductions in postprandial lipemia (Gill, et al., 2002, Tsetsonis and Hardman, 1996). An exception to this observation comes from Katsanos, et.al, (2004) where exercise at $65 \%$ of 
VO2peak was shown to be superior to exercise at $25 \%$ of $\mathrm{VO}_{2}$ peak for lowering postprandial triglycerides, despite equal energy expenditure of the exercise sessions. A lower volume of exercise appears to be sufficient to lower postprandial triglycerides when maximal or nearmaximal intensity exercise is utilized (Freese, et al., 2011). Together, these studies suggest that there may be additional benefit to performing higher intensity exercise over lower or moderateintensity exercise for lowering postprandial triglycerides.

When the energy that was expended during exercise is replaced by increasing caloric consumption through mixed-meal supplements that contain approximately $35 \%$ of calories from fat, the positive effect of exercise on lowering postprandial triglycerides is attenuated but not abolished (Burton, et al., 2008, Freese, et al., 2011). Although low-, moderate-, and highintensity exercise sessions may qualify as isocaloric, exercise of greater intensity has been shown to facilitate increased excess post-exercise oxygen consumption (EPOC) when compared to exercise of lower intensity, resulting in a greater overall energy expenditure following highintensity exercise (Borsheim and Bahr, 2003). The difference in EPOC following exercise of moderate- and high- intensity has not been quantified with the intention of determining its contribution to changes in postprandial lipemia. EPOC has been estimated when replacing the energy expenditure of exercise, yet no studies have specifically examined its individual contribution to reducing postprandial lipemia. When energy balance has been manipulated by reducing caloric consumption, postprandial triglycerides are favorably altered, however to a lesser extent when compared to an exercise induced energy deficit (Gill and Hardman, 2000, Maraki and Sidossis, 2010). It remains to be determined whether replacing the caloric expenditure incurred in EPOC affects postprandial lipemia. 
Low- intensity aerobic exercise at 35 to $45 \% \mathrm{VO}_{2}$ peak significantly lowers postprandial lipemia, while an isocaloric session of exercise at 60 to $70 \%$ of $\mathrm{VO}_{2}$ peak was shown to lower postprandial triglycerides non-significantly in men with MetS (Mestek, et al., 2008). The effects of higher-intensity exercise (at or above $70 \% \mathrm{VO}_{2}$ peak) on postprandial lipemia have not been examined in sedentary, overweight males. The purpose of this study was to evaluate the effects of low- and high-intensity exercise on postprandial lipemia in sedentary overweight men and to determine the contribution of EPOC to reductions in postprandial lipemia.

\section{MATERIALS AND METHODS}

\section{Subjects}

Participant characteristics are presented in Table 1. Middle-aged, obese and overweight men were recruited via informational flyers and e-mails. All participants were sedentary, reporting that they engaged in less than 2.5 hours per week of low to moderate physical activity and were free of cardiovascular and metabolic disease. Participants were weight stable, nonsmokers, were not taking any medication known to affect glucose or lipid metabolism, were lactose tolerant, and were free from orthopedic injury that would limit walking or jogging on a treadmill. All procedures were reviewed and approved by the Internal Review Board (IRB) at Baylor University and each participant gave written, informed consent before the study began. Prior to subject recruitment a power analysis was conducted to determine the number of participants necessary to maintain power at 0.8 at an alpha level of 0.05 . Effect sizes from studies that employed similar study design and population criteria were calculated using the 4hour triglyceride concentration values following non-exercise control and exercise interventions as primary variables of interest. The calculated effect size was 0.98 , and it was determined that 6 participants were needed for analysis. 


\section{Preliminary Screening}

A phone interview was conducted to assess the volunteer's age, physical activity habits, and disease state. Volunteers who met entry criteria visited the lab on 2 occasions thereafter. Participants completed a health-history questionnaire that was reviewed by a physician prior to exercise testing.

After an 8- to 10-hour fast a small blood sample $(17 \mathrm{ml})$ was obtained by venipuncture from an antecubital vein for the determination of baseline blood glucose and lipids (Becton Dickinson (BD) Vacutainer, Franklin Lakes, NJ, USA, SST 16 x 100 mm, 7.5 mg). Body composition was determined using dual-energy x-ray absorptiometry (DXA) (Hologic, Bedford, MA, USA). Participants performed a standardized maximal graded exercise test on a treadmill using the modified Bruce protocol to determine their cardiovascular fitness (Bruce, et al., 1973). The cardiovascular response to exercise was determined using continuous 12- lead electrocardiography (Cardio Control, Welch Allyn, Skaneateles, NY, USA). VO 2 peak was determined via respiratory gas analysis throughout the graded exercise test and was defined as the highest $\mathrm{VO}_{2}$ maintained for one minute (ParvoMedics, Sandy, UT, USA). Two of 3 criteria were required for validation of maximal effort: 1) heart rate within 10 beats of age predicted maximum; 2) rating of perceived exertion $\geq 18$, or; 3 ) respiratory exchange ratio (RER) $\geq 1.15$. The maximum heart rate and $\mathrm{VO}_{2}$ peak obtained from the participant's graded exercise test was used to determine exercise intensities that are equal to $40-50 \%$ and $70-80 \%$ of heart rate reserve (HRR) and $\mathrm{VO}_{2}$ reserve $\left(\mathrm{VO}_{2} \mathrm{R}\right)$ (Karvonen, et al., 1957). Participants who met all inclusion criteria and were cleared to exercise based on a normal cardiovascular response to exercise as reviewed by the physician were asked to continue to take part in the study. 
Participants were instructed to keep detailed records of their diet and physical activity habits for 3 days leading up to each trial. The records submitted before the initial experimental condition were replicated as closely as possible for all subsequent trials. Failure to comply with replication of dietary and physical activity habits was established a priori as an exclusion criteria due to the potential confounding influence of these variables on postprandial lipemia. Dietary intake and macronutrient composition were analyzed using nutritional analysis software (Food Processor, SLQ, Version 10.7, ESHA Research, Salem, OR, USA).

\section{Experimental Trials}

Overview

Each participant performed 4 experimental trials: non-exercise control (CON), lowintensity exercise (LI) at 40 to $50 \% \mathrm{VO}_{2} \mathrm{R}$, high-intensity exercise (HI) at 70 to $80 \% \mathrm{VO}_{2} \mathrm{R}$, and high-intensity exercise + EPOC energy replacement $(\mathrm{HI}+$ EERM). Testing order was randomized except for the fourth and final trial where the EPOC energy difference between LI and HI was replaced. The fourth trial was not randomized due to the necessity of determining the EPOC energy expenditure difference between LI and HI prior to energy replacement. Each condition was separated by at least 5 days and no more than 14 days. On the morning of each trial, the participants reported to the lab in the morning after a 12-hour fast limited to water intake only. Each was measured for height and weight (SECA, Hamburg, Germany), and fitted with a heart rate monitor (Polar, Lake Success, NY, USA). Heart rate and blood pressure were measured after 5 minutes of seated rest. All experimental trials began in the morning between approximately 7 and 9 a.m., and successive trials for each participant were standardized to begin at the same time of day. A high-fat test meal in the form of a milk shake was consumed following respiratory gas analysis in $\mathrm{CON}$ and 2 hours following each exercise session in LI, HI 
and HI + EERM. Blood samples were obtained prior to the determination of resting energy expenditure, immediately before the high-fat meal and at 2, 4 and 6 hours postprandially. Exercise Interventions

Participants sat upright and respiratory gasses were measured for 15 minutes using a portable respiratory gas analysis system $\left(\mathrm{VO}_{2000}\right.$, Medgraphics, St. Paul, MN, USA). The final 10 minutes of oxygen consumption were averaged and used for the calculation of resting caloric expenditure. Participants were then asked to walk or jog on a treadmill in order to expend 500 calories of energy. Warm-up consisted of walking for 3 minutes at 2.5 miles per hour and a $2 \%$ grade.

The approximate time needed for each session and the rate of caloric expenditure was estimated before each session using the oxygen consumption data obtained from the participant's graded exercise test and a 5 kilocalorie $(\mathrm{kcal}) * \mathrm{~L}^{-1}$ of $\mathrm{O}_{2}$ equivalent (Karvonen, et al., 1957). During the HI session participants were asked to exercise continuously at $70-80 \%$ of $\mathrm{VO}_{2} \mathrm{R}$ for approximately 45-60 minutes. During the LI session participants were asked to exercise continuously at 40-50\% $\mathrm{VO}_{2} \mathrm{R}$ for approximately 70-90 minutes. Respiratory gasses were measured regularly at approximate 10-15 minute intervals to verify oxygen consumption and to determine that a 500 calorie energy expenditure had been achieved. During both HI and LI heart rate was measured continuously.

After LI and HI, EPOC was determined from respiratory gasses measured while the participant sat quietly for 2 hours or until the participant's oxygen consumption, averaged over 10-minute intervals, reached resting values obtained prior to the exercise session. Oxygen consumption was averaged over 1-minute intervals and was used to calculate caloric expenditure. Immediately after the final HI session, participants consumed a meal with a caloric 
Exercise Intensity and Postprandial Lipemia

content equal to the difference in calories spent in the hours after the LI and HI sessions. This meal was a portioned amount of a commercially available meal bar. (Peanut Butter Power Bar ${ }^{\circledR}$ : 240 kcals, $4 \mathrm{~g}$ fat, $44 \mathrm{~g}$ carbohydrate, $9 \mathrm{~g}$ protein).

\section{Non-Exercise Control}

Participants sat upright and respiratory gasses were measured using a portable respiratory gas analysis system for 45 minutes. The final 10 minutes of resting data were averaged for the determination of resting oxygen consumption. This measurement allowed for the estimation of caloric expenditure under fasting and non-exercised conditions.

\section{Test Meal}

Participants consumed the test meal within 15 minutes of the pre-meal blood draw. The high-fat milk shake was composed of $255 \mathrm{~mL}$ of whipping cream and $74 \mathrm{~g}$ of ice cream $(1,010$ kcals, $100 \mathrm{~g}$ fat, $99 \mathrm{~g}$ saturated fat, $17 \mathrm{~g}$ carbohydrate and $3 \mathrm{~g}$ protein) (Mestek, et al., 2008, Plaisance, et al., 2008, Zhang, et al., 1998).

\section{Blood Sampling}

Blood samples were obtained prior to determination of resting energy expenditure, immediately before ingesting the high-fat test meal, and again at 2, 4, and 6 hours postprandially (BD Vacutainer, Franklin Lakes, NJ, USA, 16 x 100 mm; BD Vacutainer, Franklin Lakes, NJ, USA, 13 x 75 mm, K2EDTA). A plastic catheter (BD Vacutainer, Franklin Lakes, NJ, USA, 0.9 * $25 \mathrm{~mm}$ ) was inserted into the antecubital vein and an intermittent injection site was attached (Kawasumi Laboratories, Inc., Tokyo, Japan).

Following each blood draw sodium heparin was injected to maintain patency (Heparin Lock Flush, 10 USD units/mL, APP Pharmaceuticals, Schaumburg, IL, USA). Prior to sampling before the test meal and at 2, 4 and 6 hours, a small amount of blood was removed to ensure no 
sodium heparin contaminated the samples. At each sampling point 4 microcapillary tubes were filled with blood and centrifuged at $3900 \mathrm{X}$ g for 15 minutes to determine hematocrit and assess alterations in fluid volume (75 mm Hematocrit Tubes, Drummond, Broomall, PA, USA; ZipOcrit LW Scientific, Lawrenceville, GA, USA) (Van Beaumont, 1973). Vacutainers were allowed to clot on ice for 30 minutes before being centrifuged at $3500 \mathrm{Xg}$ for 15 minutes (Clinical 50, VWR, Randor, PA, USA). Serum and plasma were aliquoted into $2.0 \mathrm{~mL}$ plastic ultracentrifuge tubes and stored at $-80.0^{\circ} \mathrm{C}$.

\section{Sample Analyses}

Triglyceride, insulin, HDLC, non-esterified fatty acids (NEFA), non-HDLC, total cholesterol (TC), apolipoproein B (ApoB), and apolipoprotein A (ApoA) were measured from plasma and serum samples. Homeostatic model assessment (HOMA) and glucose to insulin ratio $(\mathrm{G} / \mathrm{I}$ ratio) were calculated to assess insulin resistance in the fasted state $[\mathrm{HOMA}=$ fasting glucose $(\mathrm{mg} / \mathrm{dl}) /$ fasting insulin $(\mathrm{mU} / \mathrm{mL}) * 22.5 ; \mathrm{G} / \mathrm{I}$ ratio = fasting glucose $(\mathrm{mg} / \mathrm{dl}) /$ fasting insulin concentration (mU/mL)] (Matthews, et al., 1985). Triglycerides, total cholesterol, LDLC and glucose were determined enzymatically (Siemens Vista Autoanalyzer, Malvern, PA, USA). NEFA was determined enzymatically as described by Wako Diagnostics (Wako Diagnostics, Richmond, VA, USA). The intra-assay coefficients of variation for triglycerides, total cholesterol, LDLC, glucose and NEFA were $1.5 \%, 2.5 \%, 3.1 \%, 1.8 \%$, and $2.9 \%$, respectively. HDLC was determined by immunoinhibition colorimetrically as described by Siemens (Siemens Vista Autoanalyzer, Malvern, PA, USA). The intra-assay coefficient of variation for HDLC was 3.1\%. ApoB and ApoA1 were determined by immunoinhibition, and the ApoB/A1 ratio was calculated by dividing Apo B by ApoA1. The intra-assay coefficients of variation for ApoB and ApoA1 were $2.4 \%$ and $2.6 \%$. The non-HDLC was calculated by subtracting HDLC from total 
cholesterol. Insulin was determined by enzyme linked immunosorbent assay (ELISA) (Siemens Vista Autoanalyzer, Malvern, PA, USA). The intra-assay coefficient of variation for insulin was $2.1 \%$.

\section{Statistical Analyses}

The mean triglyceride value at each time point was used to analyze postprandial changes in triglycerides. Additionally, the total $\left(\mathrm{AUC}_{\mathrm{T}}\right)$ and incremental $\left(\mathrm{AUC}_{\mathrm{I}}\right)$ areas under the curve were calculated using the trapezoidal rule and the equations detailed below (Matthews, et al., 1990). The $\mathrm{AUC}_{\mathrm{I}}$ was used to reflect the postprandial triglyceride area under the curve response while accounting for fasting triglyceride concentrations. Total and incremental areas under the curve were calculated to examine differences in insulin concentration between conditions.

$$
\begin{aligned}
& \operatorname{AUC}_{\mathrm{T}}\left(\mathrm{mmol} * \mathrm{~L}^{-1} * 6 \mathrm{~h}\right)=\mathrm{n}_{\mathrm{B}}+2\left[\mathrm{n}_{2}+\mathrm{n}_{4}\right]+\mathrm{n}_{6} \\
& \mathrm{AUC}_{\mathrm{I}}\left(\mathrm{mmol} * \mathrm{~L}^{-1} * 6 \mathrm{~h}\right)=2\left[\mathrm{n}_{2}+\mathrm{n}_{4}\right]+\mathrm{n}_{6}-5 \mathrm{n}_{\mathrm{B}}
\end{aligned}
$$

Proc Univariate procedures were performed to determine data distribution. Differences in fasting triglyceride concentrations, $\mathrm{AUC}_{\mathrm{T}}$ and $\mathrm{AUC}_{\mathrm{I}}$ were determined using separate repeated measures analysis of variance (ANOVA). Temporal alterations in insulin, HDLC, NEFA, nonHDLC, total cholesterol, ApoB, and ApoA were examined using repeated measures ANOVA's. Additionally, $\mathrm{AUC}_{\mathrm{T}}$ and $\mathrm{AUC}_{\mathrm{I}}$ were calculated to assess postprandial alterations in insulin concentrations. Follow-up was performed by using Duncan's New Multiple Range test when significant differences were observed between groups. Statistical Analysis Software (SAS, Version 9.2, Cary, NC, USA) was utilized for analysis of data and comparison wise alpha level of $p<0.05$ was considered statistically significant. 


\section{RESULTS}

\section{Dietary Intake and Fasting Physiologic Parameters}

Nine men were recruited to participate, however, after completing the study and following analysis of dietary records, it was determined that 2 participants participated in activities prior to the control trial that could not be replicated prior to the other experimental conditions and had strong potential to affect the blood lipid response to the high-fat test meal. One of these participants reported consuming a high-calorie meal that contained alcohol prior to the control trial, and the other participant reported gastrointestinal distress in the hours leading up to and during the control trial. For this reason, data is presented for the 7 individuals who were able to closely replicate dietary and physical activity habits leading up to each experimental trial. Physiologic variables across conditions are presented in Table 2. Analysis of variables in the fasted state before each of the experimental trials confirmed that participants began each trial under similar physiologic conditions. Body weight, glucose, insulin, HOMA score, G/I ratio, and resting energy expenditure were not significantly different between the experimental conditions ( $p>0.05$ for all variables). Reported intake of total calories, macronutrients, and the polyunsaturated/saturated fat ratio were not different between the 4 conditions.

\section{Responses to Treadmill Exercise}

Characteristics of each exercise session are presented in Table 3. The caloric

expenditures of the LI, HI, and HI + EERM exercise trials were each approximately 500 calories, with no significant differences between the conditions $(p=0.975)$. The exercise time for both high-intensity trials averaged $47+2$ minutes, and, by design, the mean exercise time for the LI session was significantly longer, at $74+2$ minutes $(p<0.0001)$. Participants achieved intensities of $39.1+0.6 \%$ for $\mathrm{LI}$, and $69.3+1.5$, and $70.3+2.9 \%$ of $\mathrm{VO}_{2}$ peak during the high- 
Exercise Intensity and Postprandial Lipemia

intensity trials $(p<0.0001)$. The relative exercise intensities and average RER during the highintensity trials were statistically similar, and were significantly higher than those measured in the LI trial as expected (Intensity, $p<0.0001$; RER, $p<0.001$ ). Average heart rate was significantly different between the three conditions: $112+5(\mathrm{LI}), 149+6(\mathrm{HI})$, and $140+5(\mathrm{HI}+\mathrm{EERM})(p$ $<0.0001)$. All participants were able to complete each of the exercise trials with no adverse events.

Results for EPOC measurements are presented in Table 4. Following LI and HI exercise, oxygen consumption was elevated above rest for an average of and $24+17$ and $27+16$ minutes $(p=0.119)$. EPOC was more than 2-times higher following HI when compared to LI exercise $(9.1+4.3 \mathrm{~L}$ vs. $4.4+2.0 \mathrm{~L})$, yet there was no statistically significant difference between the conditions $(p=0.098)$. The energy expenditures resulting from EPOC following HI and LI exercise were equal to $45.3+21.7$ and $22.0+10.0$ calories $(p=0.099)$. There were no statistically significant differences in EPOC time or calories expended during EPOC between exercise conditions.

\section{Postprandial and Fasting Blood Lipid Responses}

There were no statistically significant changes in plasma volume across conditions or time points, therefore, values presented are derived from unadjusted data. $(p=0.256)$.

The temporal triglyceride response is presented in Figure 1. At 4 hours, triglyceride concentrations were significantly reduced below CON values for both the LI and HI exercise trials, with no significant difference between the control and HI + EERM. Six hours after LI, a significantly reduced triglyceride response was observed when compared to CON (triglyceride by time, $p<0.0001)$. 
Triglyceride $\mathrm{AUC}_{\mathrm{I}}$ is depicted in Figure 2. For both total and incremental areas under the curve, the LI, HI, and HI + EERM trials were significantly lower when compared to the control trial $\left(\mathrm{AUC}_{\mathrm{T}}, p<0.05 ; \mathrm{AUC}_{\mathrm{I}}, p<0.05\right)$. No statistically significant differences were found for total or incremental triglyceride responses between the 3 exercise conditions.

The temporal NEFA responses to exercise are presented in Figure 3. NEFA concentrations decreased at 2 hours, and rose at hours 4 and 6 under all conditions. At 0 and 2 hours, NEFA concentrations were significantly higher during each exercise condition when compared to control.

The temporal responses of TC, HDLC, ApoB, ApoA1, and the ApoB/A1 ratio are presented in Table 5. HDLC was decreased during the postprandial period significantly at both 2 and 4-6 hours when compared to baseline $(p<0.0001)$. Apo B and the ApoB/A1 ratio rose significantly across time points as early as 2 hours into the postprandial period (ApoB, $p<$ 0.0001; ApoB/A1 ratio, $p<0.05)$. ApoA1 was significantly elevated at 4 and 6 hours postprandially $(p<0.05)$

\section{Fasting and Postprandial Glucose and Insulin Responses}

Insulin concentrations were statistically similar between the conditions, but a main effect was found for time, with the 2-hour postprandial insulin concentrations significantly higher than all other time points across conditions. Likewise, glucose levels did not differ significantly across conditions, but a significant interaction was found for time, with significantly lower values observed at 4 and 6 hours when compared 0 and 2-hour time points $(p<0.0001)$. Total and incremental areas under the curve for insulin were not significantly different between any of the 4 conditions (total, $p=0.824$; incremental, $p=0.061$ ). 
Exercise Intensity and Postprandial Lipemia

\section{DISCUSSION}

Our findings indicate that, in sedentary, overweight men, exercise of a 500 calorie energy expenditure at both 40 to $50 \%$ and 70 to $80 \%$ of $\mathrm{VO}_{2} \mathrm{R}$ is sufficient to favorably alter the postprandial hypertriglyceridemia incurred following a high fat meal. Contrary to our hypothesis, HI was not superior to LI in lowering postprandial triglycerides. Our results also demonstrate that differences in EPOC between low- and high- intensity exercise did not contribute substantially to alterations in postprandial lipemia.

Our findings are in agreement with other studies that have indicated exercise resulting in a 500-calorie energy expenditure significantly lowers postprandial triglycerides (Maraki and Sidossis, 2013). Zhang, et.al, (2007) has shown that exercise at $60 \%$ of VO2peak lowers postprandial triglycerides when 45 or 60 minutes is performed, but not 30 minutes in men with MetS. The energy expenditures of these sessions were approximately 450, 597 and 300 calories, respectively. These findings agree with our own, suggesting that exercise at 60 to $70 \%$ of VO2peak with a 450 to 500 calorie energy expenditure produces statistically significant changes in postprandial triglycerides in unfit men. Mestek, et.al, (2008) has shown that, when compared to non-exercise control, exercise resulting in a 500 calorie energy expenditure and averaging $39 \%$ of $\mathrm{VO} 2$ peak significantly lowers postprandial triglyceride $\mathrm{AUC}_{\mathrm{I}}$ by $27 \%$, while exercise at $63 \%$ lowers triglycerides similarly, although not significantly by $20 \%$ in men with MetS. Our work adds to these findings by demonstrating that exercise at a higher relative percentage of $\mathrm{VO}_{2}$ peak (69 to 70 compared to $63 \%$ of $\mathrm{VO}_{2}$ peak) results in similar reductions in postprandial triglycerides compared to low-intensity exercise (39\% of $\mathrm{VO}_{2}$ peak). In contrast to Mestek, et al., (2008), we found the reduction in postprandial triglycerides to be significant following low- and high-intensity exercise when compared to non-exercise control, with reductions in triglyceride 
$\mathrm{AUC}_{\mathrm{I}}$ of $31 \%$ following $\mathrm{LI}$ and $27 \%$ following $\mathrm{HI}$. This finding may indicate that there is, indeed, a benefit to performing high-intensity exercise. However, subtle differences in study design related to meal timing may have contributed to the disparity in our findings. Our participants ingested the high-fat meal 2 hours following exercise, while in Mestek's study approximately 12 hours separated exercise and meal ingestion.

Our findings may seem contradictory to others who have shown exercise intensity to be a factor in lowering postprandial triglycerides. Katsanos, et. al. (2004) reported finding a significantly lower triglyceride response following moderate- $(65 \%)$ when compared to lowintensity (25\%) exercise. However, in the former study physically active participants with a substantially higher $\mathrm{VO}_{2}$ peak were examined, and the intensities in the two exercise trials differed by $40 \%$ of $\mathrm{VO}_{2}$ peak (Katsanos, et al., 2004, Trombold, et al., 2013). The absolute differences in oxygen consumption attained by Katsanos, et al., between the low- and moderateintensity trials were greater than that achieved in our study.

Additional evidence supporting the use of high- versus low- or moderate- intensity exercise for lowering postprandial lipemia comes from studies that have examined near-maximal or maximal-intensity exercise (Freese, et al., 2011, Trombold, et al., 2013). While our findings may seem contrary and do not suggest a benefit to performing high- over low- intensity exercise in the context of lipid alterations, we examined continuous exercise at a lower intensity, with the high-intensity session averaging approximately $70 \%$ of $\mathrm{VO}_{2}$ peak. We maintained RER values below 1.0 for all participants during the high-intensity trial and, for multiple subjects, it was not possible to maintain a workload that elicited an exercise intensity close to $80 \%$ of $\mathrm{VO}_{2}$ peak without increasing the RER to at or near 1.0. Maintaining a continuous intensity of aerobic exercise in order to expend the threshold (450 to 500) number of calories required to positively 
affect postprandial lipemia at intensity higher than $70 \%$ may not be possible for many untrained subjects. Because a lower volume of exercise may be sufficient to lower postprandial triglycerides when exercise is near maximal intensity, the effects of maximal or near maximal interval exercise on postprandial lipemia in overweight males should be determined.

Although the differences were not statistically significant, EPOC was 210\% higher following HI when compared to LI, increasing from 9.1 to $4.4 \mathrm{~L}$. These findings are similar to others reported in the literature (Gore and Withers, 1990, Phelain, et al., 1997). Borsheim and Bahr (2003) have conducted an extensive review of the literature on EPOC and have concluded that exercise intensity makes the greatest contribution to EPOC. Our participants were of low cardiovascular fitness, with an average $\mathrm{VO}_{2}$ peak of $31.1+7.5$, representing the $10^{\text {th }}$ percentile for men between the ages of 40 and 49 , and thus the absolute $\mathrm{VO}_{2}$ that each participant was able to maintain continuously during HI was relatively low compared to those of average or highfitness (Pescatello, 2013). Thus, HI for these participants may have produced a smaller EPOC than would have been observed for an individual capable of maintaining a higher oxygen consumption continuously. Although EPOC is indeed elevated to a greater extent following HI when compared to LI exercise, the differences are not robust enough to drastically increase energy expenditure at the intensities utilized.

Re-feeding the caloric difference that resulted from EPOC between low- and highintensity exercise did not significantly affect the ability of exercise to positively impact postprandial lipemia. Three previous studies have shown that, when the energy that was expended during exercise is fully replaced by increasing caloric intake, the positive effects of exercise on lowering postprandial lipemia is significantly lessened but not abolished (Burton, et al., 2008, Freese, et al., 2011, Harrison, et al., 2009). Our work adds to these findings by 
demonstrating that the caloric expenditure of EPOC alone is not sufficient to affect postprandial triglycerides when exercise is performed at 39 and $70 \%$ of $\mathrm{VO}_{2}$ peak. We re-fed a small meal to our participants, with a mean of 23.2 calories, compared with approximately $670,1,500$ and 260 calories in the previously mentioned re-feeding studies (Burton, et al., 2008, Freese, et al., 2011, Harrison, et al., 2009).The caloric threshold at which re-feeding negates the positive effect of exercise energy expenditure on postprandial triglycerides remains to be determined.

While the decrements in postprandial lipemia appear to be mediated by energy expenditure and energy intake, the precise mechanisms responsible remain elusive and were not directly investigated in this study. Likely candidates include reduced hepatic VLDL secretion and increased lipoprotein lipase (LPL) activity (Dekker, et al., 2010). LPL activity has been shown to be increased from 4 to 24 hours following exercise at 60 to $75 \%$ of $\mathrm{VO}_{2}$ peak (Greiwe, et al., 2000, Kiens, et al., 1989, Nilsson-Ehle, et al., 1980). In obese men, moderate exercise performed the day before a high-fat meal results in increased clearance of VLDL particles when compared to non-exercise control (Al-Shayji, et al., 2012). In addition, a 500 calorie energy expenditure results in increased clearance of VLDL particles in addition to decreased hepatic VLDL production in women (Bellou, et al., 2012). It is likely that increased triglyceride clearance and/or reduced hepatic VLDL secretion contributed to our findings.

Prior moderate-intensity exercise has been shown to lower postprandial insulin concentrations, a finding that we did not observe (Katsanos, et al., 2004). While decreased insulin concentration is associated with increased skeletal muscle LPL activity, others have reported reductions in postprandial lipemia in the absence of reduced insulin concentration (Kiens, et al., 1989, Mestek, et al., 2008). Our test meal was relatively low in carbohydrate (17 grams) and consisted primarily of fatty acids. It is possible that the insulin response would have 
differed had the meal had higher carbohydrate content. Although reduced insulin concentration may be permissive in allowing increased LPL activity, increased post-heparin LPL activity has been observed in the absence of significantly reduced insulin levels and postprandial triglycerides have been shown to be lowered even in the absence of significant increases in muscle LPL activity (Herd, et al., 2001, Katsanos, et al., 2004).

While the CON, LI and HI trials were completed in randomized order, because of our research questions it was not possible to randomize the fourth and final exercise condition. It was necessary for participants to complete both exercise trials so that EPOC energy expenditure could be determined and the difference in caloric expenditure during EPOC following LI and HI replaced. We do not believe that the inability to randomize the re-feeding trials has bearing on our findings, as the HI + EERM trial was identical to the first high-intensity exercise session completed, and caloric expenditure between the 3 exercise conditions was statistically similar. In conclusion, we found that continuous exercise at 39 and $69-70 \%$ of VO2peak significantly and similarly lowers postprandial triglycerides following a high fat meal in sedentary, overweight men. Our results indicate that EPOC does not make a primary contribution to the favorable effects of exercise on reducing postprandial lipemia. Low- or high- intensity exercise can be recommended to sedentary individuals for reducing postprandial triglycerides.

\section{Disclaimer}

The authors report no conflicts of interest associated with this manuscript. 


\section{REFERENCES}

Al-Shayji, I. A., Caslake, M. J. and Gill, J. M. 2012. Effects of moderate exercise on VLDL(1) and Intralipid kinetics in overweight/obese middle-aged men. Am. J. Physiol. Endocrinol. Metab, 302 (3): E349-55. 10.1152/ajpendo.00498.2011.

Aldred, H. E., Perry, I. C. and Hardman, A. E. 1994. The effect of a single bout of brisk walking on postprandial lipemia in normolipidemic young adults. Metabolism, 43 (7): 836-41.

Bansal, S., Buring, J. E., Rifai, N., Mora, S., Sacks, F. M. and Ridker, P. M. 2007. Fasting compared with nonfasting triglycerides and risk of cardiovascular events in women. JAMA, 298 (3): 309-16. 10.1001/jama.298.3.309.

Bellou, E., Siopi, A., Galani, M., Maraki, M., Tsekouras, Y. E., Panagiotakos, D. B., Kavouras, S. A., Magkos, F. and Sidossis, L. S. 2012. Acute Effects of Exercise and Calorie Restriction on Triglyceride Metabolism in Women. Med. Sci. Sports. Exerc, 10.1249/MSS.0b013e318278183e.

Borsheim, E. and Bahr, R. 2003. Effect of exercise intensity, duration and mode on post-exercise oxygen consumption. Sports Med, 33 (14): 1037-60.

Bruce, R. A., Cooper, M. N., Gey, G. O., Fisher, L. D. and Peterson, D. R. 1973. Variations in responses to maximal exercise in health and in cardiovascular disease. Angiology, 24 (11): 691-702.

Burton, F. L., Malkova, D., Caslake, M. J. and Gill, J. M. 2008. Energy replacement attenuates the effects of prior moderate exercise on postprandial metabolism in overweight/obese men. Int. J. Obes. (Lond), 32 (3): 481-9. 10.1038/sj.ijo.0803754. 
Exercise Intensity and Postprandial Lipemia

Dekker, M. J., Graham, T. E., Ooi, T. C. and Robinson, L. E. 2010. Exercise prior to fat ingestion lowers fasting and postprandial VLDL and decreases adipose tissue IL-6 and GIP receptor mRNA in hypertriacylglycerolemic men. J. Nutr. Biochem, 21 (10): 983-90. 10.1016/j.jnutbio.2009.08.004.

Freese, E. C., Levine, A. S., Chapman, D. P., Hausman, D. B. and Cureton, K. J. 2011. Effects of acute sprint interval cycling and energy replacement on postprandial lipemia. J. Appl. Physiol, 111 (6): 1584-9. 10.1152/japplphysiol.00416.2011.

Gill, J. M., Herd, S. L. and Hardman, A. E. 2002. Moderate exercise and post-prandial metabolism: issues of dose-response. J. Sports Sci, 20 (12): 961-7. $10.1080 / 026404102321011715$.

Gill, J. M. and Hardman, A. E. 2000. Postprandial lipemia: effects of exercise and restriction of energy intake compared. Am. J. Clin. Nutr, 71 (2): 465-71.

Gill, J. M., Murphy, M. H. and Hardman, A. E. 1998. Postprandial lipemia: effects of intermittent versus continuous exercise. Med. Sci. Sports Exerc, 30 (10): 1515-20.

Gore, C. J. and Withers, R. T. 1990. The effect of exercise intensity and duration on the oxygen deficit and excess post-exercise oxygen consumption. Eur. J. Appl. Physiol. Occup. Physiol, 60 (3): 169-74.

Greiwe, J. S., Holloszy, J. O. and Semenkovich, C. F. 2000. Exercise induces lipoprotein lipase and GLUT-4 protein in muscle independent of adrenergic-receptor signaling. J. Appl. Physiol, 89 (1): 176-81. 
Harrison, M., O'Gorman, D. J., McCaffrey, N., Hamilton, M. T., Zderic, T. W., Carson, B. P. and Moyna, N. M. 2009. Influence of acute exercise with and without carbohydrate replacement on postprandial lipid metabolism. J. Appl. Physiol, 106 (3): 943-9. 10.1152/japplphysiol.91367.2008.

Herd, S. L., Kiens, B., Boobis, L. H. and Hardman, A. E. 2001. Moderate exercise, postprandial lipemia, and skeletal muscle lipoprotein lipase activity. Metabolism, 50 (7): 756-62. 10.1053/meta.2001.24199.

Karpe, F., Hellenius, M. L. and Hamsten, A. 1999. Differences in postprandial concentrations of very-low-density lipoprotein and chylomicron remnants between normotriglyceridemic and hypertriglyceridemic men with and without coronary heart disease. Metabolism, 48 (3): $301-7$.

Karvonen, M. J., Kentala, E. and Mustala, O. 1957. The effects of training on heart rate; a longitudinal study. Ann. Med. Exp. Biol. Fenn, 35 (3): 307-15.

Kathiresan, S., Otvos, J. D., Sullivan, L. M., Keyes, M. J., Schaefer, E. J., Wilson, P. W., D'Agostino, R. B., Vasan, R. S. and Robins, S. J. 2006. Increased small low-density lipoprotein particle number: a prominent feature of the metabolic syndrome in the Framingham Heart Study. Circulation, 113 (1): 20-9.

10.1161/CIRCULATIONAHA.105.567107.

Katsanos, C. S., Grandjean, P. W. and Moffatt, R. J. 2004. Effects of low and moderate exercise intensity on postprandial lipemia and postheparin plasma lipoprotein lipase activity in physically active men. J. Appl. Physiol, 96 (1): 181-8. 10.1152/japplphysiol.00243.2003. 
Exercise Intensity and Postprandial Lipemia

Kiens, B., Lithell, H., Mikines, K. J. and Richter, E. A. 1989. Effects of insulin and exercise on muscle lipoprotein lipase activity in man and its relation to insulin action. J. Clin. Invest, 84 (4): 1124-9. 10.1172/JCI1 14275.

Kolovou, G. D., Daskalova, D., Iraklianou, S. A., Adamopoulou, E. N., Pilatis, N. D., Hatzigeorgiou, G. C. and Cokkinos, D. V. 2003. Postprandial lipemia in hypertension. J. Am. Coll. Nutr, 22 (1): 80-7.

Kolovou, G. D., Mikhailidis, D. P., Kovar, J., Lairon, D., Nordestgaard, B. G., Ooi, T. C., PerezMartinez, P., Bilianou, H., Anagnostopoulou, K. and Panotopoulos, G. 2011. Assessment and clinical relevance of non-fasting and postprandial triglycerides: an expert panel statement. Curr. Vasc. Pharmacol, 9 (3): 258-70.

Kolovou, G. D., Mikhailidis, D. P., Nordestgaard, B. G., Bilianou, H. and Panotopoulos, G. 2011. Definition of postprandial lipaemia. Curr Vasc Pharmacol. 9 (3): 292-301.

Maraki, M. and Sidossis, L. S. 2010. Effects of energy balance on postprandial triacylglycerol metabolism. Curr. Opin. Clin. Nutr. Metab. Care, 13 (6): 608-17. 10.1097/MCO.0b013e32833flaae.

Maraki, M. I. and Sidossis, L. S. 2013. The latest on the effect of prior exercise on postprandial lipaemia. Sports Med, 43 (6): 463-81. 10.1007/s40279-013-0046-9.

Matthews, D. R., Hosker, J. P., Rudenski, A. S., Naylor, B. A., Treacher, D. F. and Turner, R. C. 1985. Homeostasis model assessment: insulin resistance and beta-cell function from fasting plasma glucose and insulin concentrations in man. Diabetologia, 28 (7): 412-9.

Matthews, J. N., Altman, D. G., Campbell, M. J. and Royston, P. 1990. Analysis of serial measurements in medical research. BMJ, 300 (6719): 230-5. 
Mestek, M. L., Plaisance, E. P., Ratcliff, L. A., Taylor, J. K., Wee, S. O. and Grandjean, P. W. 2008. Aerobic exercise and postprandial lipemia in men with the metabolic syndrome. Med. Sci. Sports. Exerc, 40 (12): 2105-11. 10.1249/MSS.0b013e3181822ebd.

Nilsson-Ehle, P., Garfinkel, A. S. and Schotz, M. C. 1980. Lipolytic enzymes and plasma lipoprotein metabolism. Annu. Rev. Biochem, 49 667-93.

10.1146/annurev.bi.49.070180.003315.

Nordestgaard, B. G., Benn, M., Schnohr, P. and Tybjaerg-Hansen, A. 2007. Nonfasting triglycerides and risk of myocardial infarction, ischemic heart disease, and death in men and women. JAMA, 298 (3): 299-308. 10.1001/jama.298.3.299.

Park, S. H., Kim, J. Y., Lee, J. H. and Park, H. Y. 2011. Elevated oxidized low-density lipoprotein concentrations in postmenopausal women with the metabolic syndrome. Clin. Chim. Acta, 412 (5-6): 435-40. 10.1016/j.cca.2010.11.017.

Patsch, J. R., Miesenbock, G., Hopferwieser, T., Muhlberger, V., Knapp, E., Dunn, J. K., Gotto, A. M., Jr. and Patsch, W. 1992. Relation of triglyceride metabolism and coronary artery disease. Studies in the postprandial state. Arterioscler. Thromb, 12 (11): 1336-45.

Pescatello, L. S., Arena, R., Reibe, D., Thompson, P.D. 2013. ACSM's Guidelines for Exercise Testing \& Prescription. Lippincott, Williams and Wilkins, Champaign, Il.

Petitt, D. S. and Cureton, K. J. 2003. Effects of prior exercise on postprandial lipemia: a quantitative review. Metabolism, 52 (4): 418-24. 10.1053/meta.2003.50071.

Phelain, J. F., Reinke, E., Harris, M. A. and Melby, C. L. 1997. Postexercise energy expenditure and substrate oxidation in young women resulting from exercise bouts of different intensity. J. Am. Coll. Nutr, 16 (2): 140-6. 
Exercise Intensity and Postprandial Lipemia

Plaisance, E. P., Mestek, M. L., Mahurin, A. J., Taylor, J. K., Moncada-Jimenez, J. and Grandjean, P. W. 2008. Postprandial triglyceride responses to aerobic exercise and extended-release niacin. Am. J. Clin. Nutr, 88 (1): 30-7.

Trombold, J. R., Christmas, K. M., Machin, D. R., Kim, I. Y. and Coyle, E. F. 2013. Acute highintensity endurance exercise is more effective than moderate-intensity exercise for attenuation of postprandial triglyceride elevation. J. Appl. Physiol, (1985). 114 (6): 792 800. 10.1152/japplphysiol.01028.2012.

Tsetsonis, N. V. and Hardman, A. E. 1996. Reduction in postprandial lipemia after walking: influence of exercise intensity. Med. Sci. Sports. Exerc, 28 (10): 1235-42.

Van Beaumont, W. 1973. Red cell volume with changes in plasma osmolarity during maximal exercise. J. Appl. Physiol, 35 (1): 47-50.

Zhang, J. Q., Ji, L. L., Nunez, G., Feathers, S., Hart, C. L. and Yao, W. X. 2004. Effect of exercise timing on postprandial lipemia in hypertriglyceridemic men. Can. J. Appl. Physiol, 29 (5): 590-603.

Zhang, J. Q., Thomas, T. R. and Ball, S. D. 1998. Effect of exercise timing on postprandial lipemia and HDL cholesterol subfractions. J. Appl. Physiol, 85 (4): 1516-22.

Zilversmit, D. B. 1995. Atherogenic nature of triglycerides, postprandial lipidemia, and triglyceride-rich remnant lipoproteins. Clin. Chem, 41 (1): 153-8. 
Exercise Intensity and Postprandial Lipemia

Table 1. Baseline anthropometric and physiological characteristics

\begin{tabular}{lccc}
\hline \multicolumn{1}{c}{ Variable } & Mean $\pm \mathrm{SD}$ & Minimum & Maximum \\
\hline Age $(\mathrm{yrs})$ & $43 \pm 10$ & 28 & 55 \\
Height $(\mathrm{m})$ & $1.77 \pm 0.06$ & 1.70 & 1.87 \\
Weight $(\mathrm{kg})$ & $100.6 \pm 17.7$ & 78.2 & 118.7 \\
BMI $\left(\mathrm{kg} / \mathrm{m}^{2}\right)$ & $31.8 \pm 4.5$ & 25.6 & 36.6 \\
Body Fat $(\%)$ & $30 \pm 6$ & 24 & 41 \\
Waist $(\mathrm{cm})$ & $107.2 \pm 14.9$ & 81.3 & 120.7 \\
SBP $(\mathrm{mmHg})$ & $128 \pm 15$ & 114 & 158 \\
DBP $(\mathrm{mmHg})$ & $81 \pm 9$ & 70 & 100 \\
VO 2 peak $(\mathrm{L} / \mathrm{min})$ & $2.9 \pm 0.3$ & 2.52 & 3.16 \\
VO 2 peak $(\mathrm{ml} / \mathrm{kg} / \mathrm{min})$ & $31.1 \pm 7.5$ & 21.4 & 40.4 \\
Glucose $(\mathrm{mmol} / \mathrm{L})$ & $5.44 \pm 0.28$ & 4.94 & 5.88 \\
Triglycerides $(\mathrm{mmol} / \mathrm{L})$ & $1.81 \pm 0.95$ & 0.70 & 3.29 \\
Total Cholesterol $(\mathrm{mmol} / \mathrm{L})$ & $4.38 \pm 0.88$ & 3.50 & 5.67 \\
HDLC $(\mathrm{mmol} / \mathrm{L})$ & $1.04 \pm 0.34$ & 0.62 & 1.48 \\
LDLC $(\mathrm{mmol} / \mathrm{L})$ & $2.51 \pm 0.60$ & 1.86 & 3.44 \\
NHDLC $(\mathrm{mmol} / \mathrm{L})$ & $3.34 \pm 0.80$ & 2.38 & 4.45
\end{tabular}

Note: Values are presented as means \pm SEM along with minimum and maximum values. BMI, body mass index; SBP, systolic blood pressure; DBP, diastolic blood pressure; HDLC, highdensity lipoprotein cholesterol; LDLC, low-density lipoprotein cholesterol; NHDLC, non-highdensity lipoprotein cholesterol. 
Exercise Intensity and Postprandial Lipemia

Table 2. Physiologic Variables Across Conditions

\begin{tabular}{lcccc}
\hline \multicolumn{1}{c}{ Variable } & CON & LI & HI & HI + EERM \\
\hline Weight $(\mathrm{kg})$ & $97.7 \pm 7.1$ & $100.1 \pm 6.5$ & $100.3 \pm 6.5$ & $100.5 \pm 6.7$ \\
REE $(\mathrm{L} / \mathrm{min})$ & $0.235 \pm 0.02$ & $0.232 \pm 0.02$ & $0.262 \pm 0.01$ & $0.251 \pm 0.02$ \\
Glucose $(\mathrm{mmol} / \mathrm{L})$ & $5.38 \pm 0.17$ & $5.88 \pm 0.06$ & $5.77 \pm 0.11$ & $5.77 \pm 0.11$ \\
Insulin $(\mathrm{mU} / \mathrm{L})$ & $15.2 \pm 3.6$ & $16.0 \pm 4.4$ & $17.4 \pm 4.4$ & $15.9 \pm 4.1$ \\
TG $(\mathrm{mmol} / \mathrm{L})$ & $1.86 \pm 0.34$ & $1.71 \pm 0.25$ & $1.92 \pm 0.24$ & $1.85 \pm 0.42$ \\
NEFA $(\mathrm{mEq} / \mathrm{L})$ & $0.377 \pm 0.05$ & $0.469 \pm 0.08$ & $0.450 \pm 0.07$ & $0.446 \pm 0.05$ \\
NHDLC $(\mathrm{mmol} / \mathrm{L})$ & $3.24 \pm 0.26$ & $3.29 \pm 0.31$ & $3.32 \pm 0.26$ & $3.21 \pm 0.28$ \\
HOMA & $3.86 \pm 0.90$ & $4.18 \pm 1.13$ & $4.49 \pm 1.11$ & $4.09 \pm 1.08$ \\
G/I ratio & $8.46 \pm 1.62$ & $9.56 \pm 2.55$ & $8.03 \pm 1.51$ & $8.91 \pm 1.73$ \\
& & & & \\
\hline
\end{tabular}

Note: Values are presented as means \pm SEM. REE, resting energy expenditure; HOMA, homeostatic model assessment, fasting glucose $(\mathrm{mg} / \mathrm{dl}) /$ fasting insulin $(\mathrm{mU} / \mathrm{mL}) * 22.5 ; \mathrm{G} / \mathrm{I}$ ratio; GIR, glucose/insulin ratio, fasting glucose $(\mathrm{mg} / \mathrm{dl}) /$ fasting insulin concentration $(\mathrm{mU} / \mathrm{mL})$. 
Table 3. Exercise Session Data

\begin{tabular}{lccc}
\hline Variable & LI & HI & HI + EERM \\
\hline Energy Expenditure (Kcal) & $500.8 \pm 0.6$ & $500.4 \pm 0.6$ & $502.4 \pm 11.5$ \\
Time $(\mathrm{min})$ & $74 \pm 2$ & $47 \pm 2^{*}$ & $47 \pm 2^{*}$ \\
Avg $\mathrm{VO}_{2}(\mathrm{ml} / \mathrm{kg} / \mathrm{min})$ & $13.8 \pm 1.0$ & $21.6 \pm 1.6^{*}$ & $22.0 \pm 2.0^{*}$ \\
$\%$ of $\mathrm{VO}_{2}$ peak & $39.1 \pm 0.6$ & $69.3 \pm 1.5^{*}$ & $70.3 \pm 2.9^{*}$ \\
Avg $\mathrm{HR}(\mathrm{bpm})$ & $112.1 \pm 5.3$ & $148.9 \pm 5.5^{*}$ & $140.4 \pm 5.2^{* \dagger}$ \\
Avg RER & $0.83 \pm 0.02$ & $0.89 \pm 0.01^{*}$ & $0.88 \pm 0.02^{*}$
\end{tabular}

Note: Values are presented as means \pm SEM. Values with similar superscripts are statistically similar. * Significantly different from LI. $\dagger$ Significantly different from HI. 
Exercise Intensity and Postprandial Lipemia

Table 4. Characteristics of EPOC Measures

\begin{tabular}{rcccc}
\hline & Mean & Min & Max & Range \\
\hline Low-Intensity & & & & 119 \\
EPTM (min) & $24 \pm 17$ & 1 & 120 & 14.9 \\
EPOC (L) & $4.4 \pm 2.0$ & 1.4 & 81.4 & 74.2 \\
EPOC Kcals & $22.1 \pm 10.0$ & 7.2 & & 117 \\
High-Intensity & & & 120 & 31.7 \\
EPTM (min) & $27 \pm 16$ & 3 & 35.1 & 158.5 \\
EPOC (L) & $9.1 \pm 4.3$ & 3.4 & 175.4 & \\
EPOC Kcals & $45.3 \pm 21.7$ & 16.9 & &
\end{tabular}

Note: Values are presented as mean \pm SEM along with minimum and maximum values. Max, maximum value; Min, minimum value; EPTM, EPOC time; EPOC Kcals, EPOC energy expenditure above rest. 
Table 5. Temporal changes in blood lipid variables

\begin{tabular}{lcccc}
\hline Variable & $0-\mathrm{hr}$ & $2-\mathrm{hr}$ & $4-\mathrm{hr}$ & 6-hr \\
\hline TC $(\mathrm{mmol} / \mathrm{L})$ & $4.22 \pm 0.13$ & $4.20 \pm 0.13$ & $4.14 \pm 0.13$ & $4.22 \pm 0.13$ \\
HDLC $(\mathrm{mmol} / \mathrm{L})$ & $0.98 \pm 0.05^{\mathrm{a}}$ & $0.93 \pm 0.05^{\mathrm{b}}$ & $0.88 \pm 0.05^{\mathrm{c}}$ & $0.88 \pm 0.05^{\mathrm{c}}$ \\
ApoB $(\mathrm{g} / \mathrm{L})$ & $0.88 \pm 0.03^{\mathrm{a}}$ & $0.96 \pm 0.4^{\mathrm{a}, \mathrm{b}}$ & $0.99 \pm 0.04^{\mathrm{c}}$ & $0.98 \pm 0.04^{\mathrm{c}}$ \\
ApoA1 $(\mathrm{g} / \mathrm{L})$ & $1.32 \pm 0.04^{\mathrm{a}}$ & $1.33 \pm 0.4^{\mathrm{a}}$ & $1.36 \pm 0.04^{\mathrm{b}}$ & $1.36 \pm 0.04^{\mathrm{b}}$ \\
ApoB/A & $0.70 \pm 0.03^{\mathrm{a}}$ & $0.73 \pm 0.03^{\mathrm{b}}$ & $0.74 \pm 0.04^{\mathrm{b}}$ & $0.74 \pm 0.04^{\mathrm{b}}$
\end{tabular}

Note: Values are presented as means \pm SEM. Means with similar letters are statistically similar. TC, total cholesterol; HDLC, high-density lipoprotein cholesterol; ApoB/A ratio, ratio of Apo B/ Apo A1. 
Exercise Intensity and Postprandial Lipemia

\section{FIGURE LEGENDS}

Fig. 1. Means + SEM for the temporal triglyceride response for control $(\diamond)$, low-intensity $(\square)$, high-intensity $(\bullet)$, and high-intensity $+\operatorname{EERM}(\boldsymbol{\Delta}) . *=$ low condition is significantly lower than control. $\dagger=$ high condition is significantly lower than control. All values were increased significantly at 2-hr when compared to baseline.

Fig. 2. Means + SEM for the incremental triglyceride area under the curve response for control (grey), low-intensity (black), high-intensity (diagonal hatch) and high-intensity + EERM (striped). $*$ = significantly different from control.

Fig. 3. Means + SEM are presented for the temporal NEFA response control $(\diamond)$, low-intensity (D), high-intensity $(\bullet)$, and high-intensity $+\operatorname{EERM}(\mathbf{\Delta}) . *$ indicates significantly difference from control, $p<0.001$. 
Exercise Intensity and Postprandial Lipemia

\section{FIGURES}

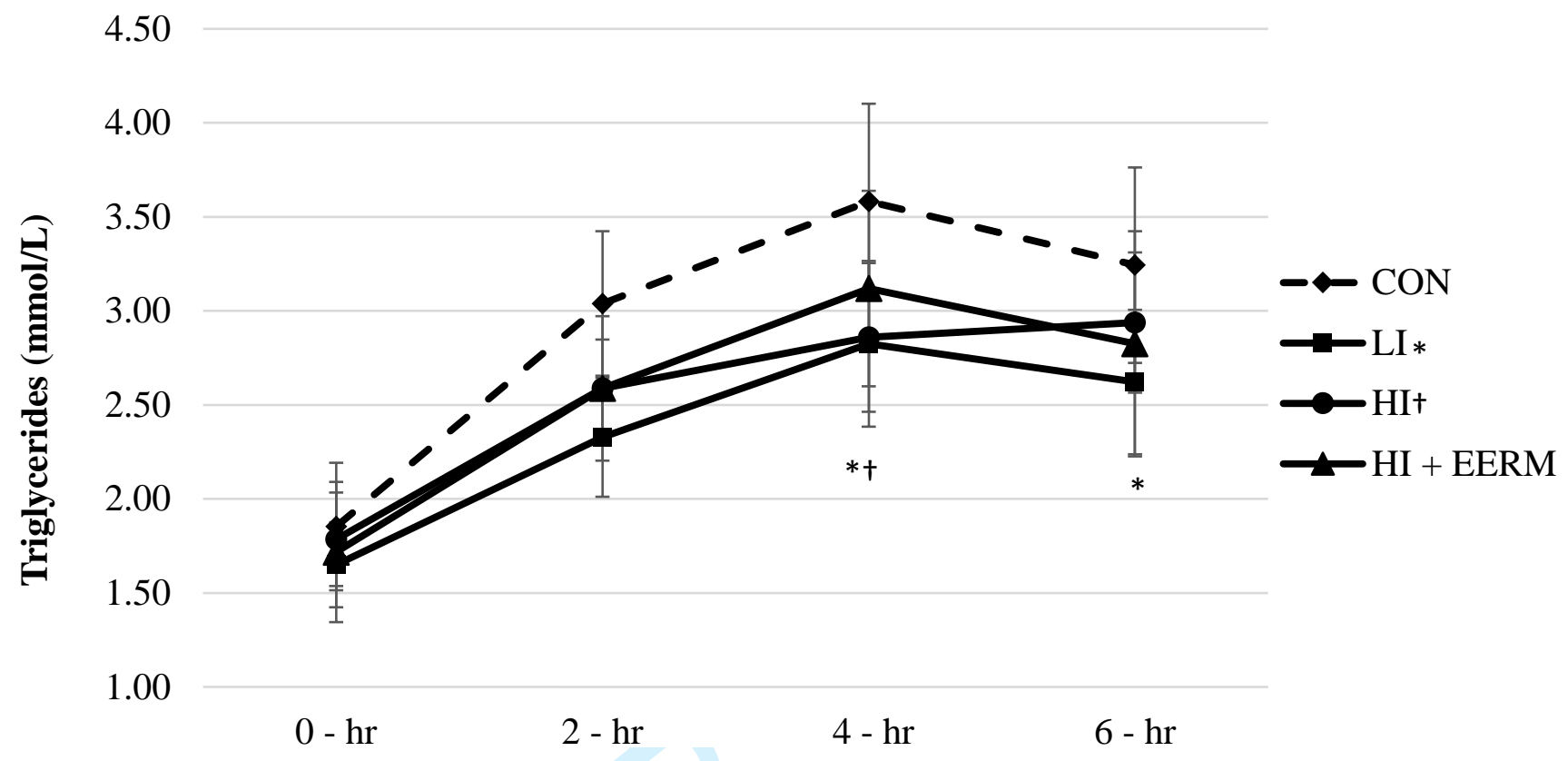

Fig. 1. 
Exercise Intensity and Postprandial Lipemia

12.00

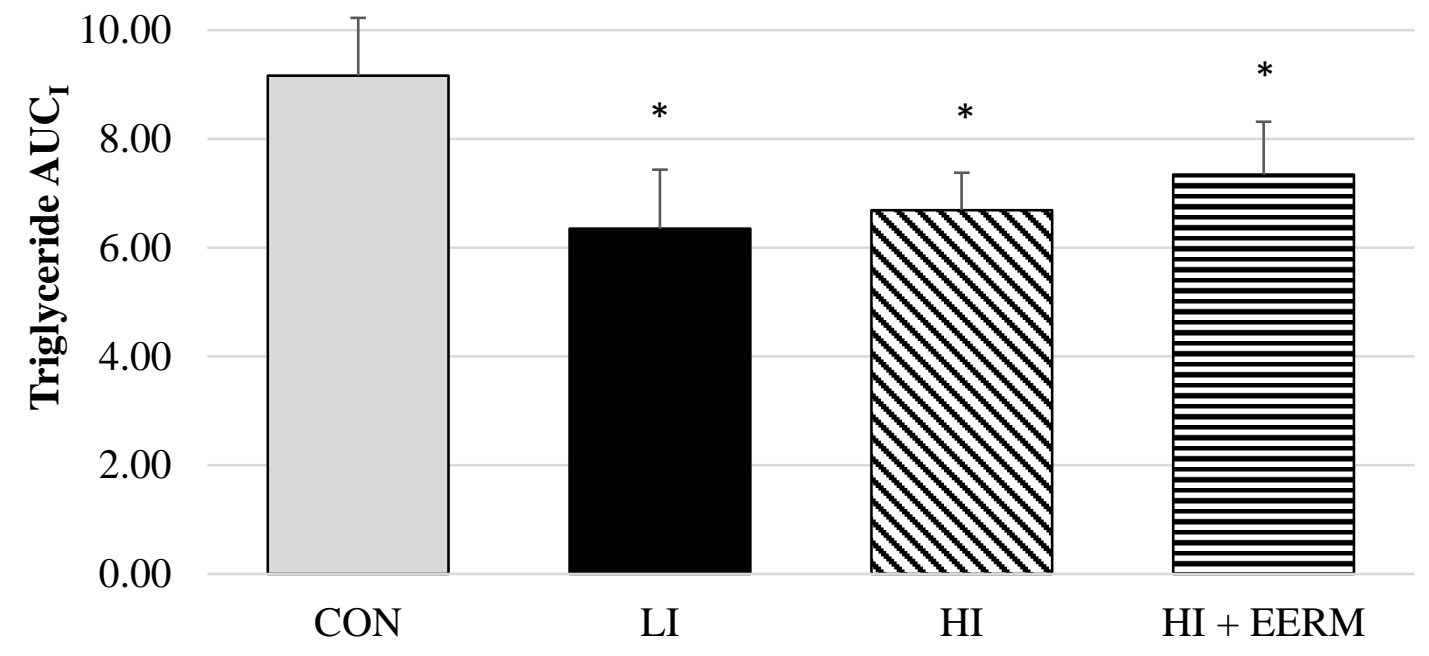

Fig. 2. 
Exercise Intensity and Postprandial Lipemia

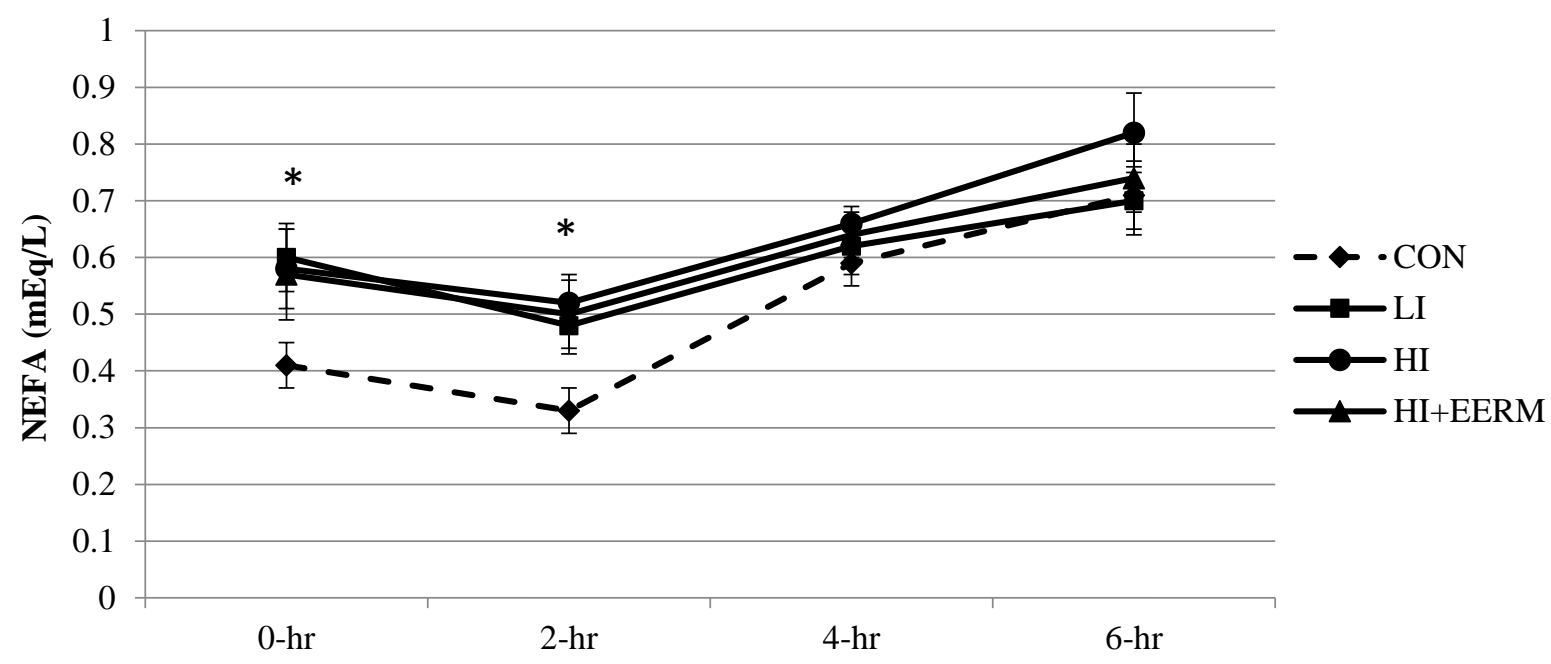

Fig. 3. 